\title{
Design for Networked Control Systems Based on Multi-Rate Technique
}

\author{
Guotao Hui*, Wanwei Li and Yingchun Wang
}

\begin{abstract}
College of Information Science and Engineering, Northeastern University, Shenyang, Liaoning, 110819, P.R. China International Cooperation and Exchange Department, Northeastern University, Shenyang, Liaoning, 110819, P.R. China College of Information Science and Engineering, Northeastern University, Shenyang, Liaoning, 110819, P.R. China
\end{abstract}

\begin{abstract}
This paper is concerned with the design of networked control systems based on multi-rata technique. Firstly, based on the Fourier series of periodic signal, a novel nonlinear two part periodic function (NTPPF) method is proposed to construct the generalized sampled-data hold function (GSHF). Secondly, a hybrid nonlinear delay system model is constructed by establishing the relationship between the network-induced delay and the frequency of the NTPPF. Furthermore, by using the Lyapunov theory, the stability condition can be obtained. Finally, a numerical example is provided to demonstrate the effectiveness and less conservativeness of the proposed methods.
\end{abstract}

Keywords: Sampled-data control system, Networked control systems (NCSs), GSHF, input delay, linear matrix inequality (LMI).

\section{INTRODUCTION}

During the last decades, the discrete-time control of continuous-time system (also called sampled-data control system) has become the focus of study due to their attractive advantages in practical application, such as the simple structure, convenient debug, high accuracy, and so on, [1, 2]. Generally, a typical sampled-data control system consists of a sampler, a digital controller which exists in the feedback channel, and a zero-order hold $(\mathrm{ZOH})$. Many design approaches for implementing different control objectives have been the subject of concern for many years $[3,4]$.

Unlike the typical sampled-data control system with $\mathrm{ZOH}$, a novel sampled-data control system with GSHF was proposed in [5]. In [6-8], the author pointed out that by using GSHF, the more efficiency of state feedback was obtained without the requirement of state estimation. Despite existing results are made to design the GSHF for sampled-data control system with the desired performance, the input delay which existed in between the sampler and GSHF, was not considered in their analysis. The design of networked control systems with piecewise constant generalized sampled-data hold function (PCGSHF) was firstly studied in [9]. They showed that the study of the delay in the feedback channel was more significant in actual applications. Moreover, in [9], the authors firstly pointed out that there are many remarkable advantages for employing PCGSHF in lieu of ZOH. The main reason of these advantages is the hold function itself which is the design variable instead of a given constant (such as $\mathrm{ZOH}$ ). Correspondingly, the additional design degree-offreedom can be gained for improving the system performance. The study in this paper can be considered as the development of the theory in [9]. It is well known that PCGSHF is a special case of GSHF. How to design the

\footnotetext{
*Address correspondence to this author at the College of Information Science and Engineering, Northeastern University, Shenyang, Liaoning, 110819, P.R. China; Tel: 13898863791; E-mail: huiguotao@vip.163.com
}

GSHF for ensuring stability of the NCSs, that is the one motivation of our present study.

Currently, the common strategy for designing GSHF in existing results depended on the Gram matrix, which was derived from the controllability of system, for instance, see the equations (8) and (9) in [10] and the similar equations in $[7,8]$. By using Gram matrix, the sampled behavior can be represented by using a time-invariant matrix, which is determined for satisfying different control objectives. Obviously, this strategy has the advantage in design of GSHF when only the period behavior is considered. However, when the network-induced delay is considered, there exist one main weakness for employing this strategy: For every time-variant input delay, it is necessary to construct a Gram matrix to determine a time-invariant matrix. But because there exists a delay-dependent relationship between any two time-invariant matrices, the determinations of them are very difficult, and even hardly achieved. To the best of our knowledge, there is still not an effective method to design the GSHF for the NCSs. That is the other one motivation of our present study.

In this paper, the design problem of NCSs based on multi-rata technique is further considered. The obtained results can be considered as the development of the theory in [9], which only consider the special case of GSHF. Firstly, based on the Fourier series of periodic signal, a novel NTPPF method is proposed to construct the GSHF. By establishing the relationship between the network-induced delay and the frequency of the NTPPF, we can obtain a hybrid nonlinear delay system model. Furthermore, the stability condition can be obtained by using the Lyapunov theory, and the NTPPF for ensuring the stability of NCSs can be designed. Lastly, a numerical example is provided to demonstrate the effectiveness of the proposed methods [11-14].

\section{PRELIMINARIES}

Consider a LTI plant of NCSs with the following statespace representation: 
$\dot{x}(t)=A x(t)+B u(t)$

where $x(t) \in \mathfrak{R}^{n}, u(t) \in \mathfrak{R}^{p}$ are the state and input of the system respectively. The pair $(A, B)$ is controllable. The control law being designed has the following form :

$u(t)=F(t-k h) x(k h)$

$(k h \leq t<(k+1) h, k \in Z)$

where $h$ and $F(t+h)=F(t)$. Here, a new GSHF $F(t)$ based on Fourier series form is proposed as follows:

$F(t)=F_{\delta}(t),(k h \leq t<(k+1) h)$

where $\delta=1,2, \mathrm{~L} q \cdot q-1$ is the number of network-induced delay. The function $F_{\delta}(t)$ is defined as follows:

$F_{\delta}(t)=\sum_{i=1}^{p \times n} a_{i}^{\delta} F_{i}^{\delta}+\sum_{i=1}^{p \times n}\left(b_{i}^{\delta} \sin \left(\frac{2 \pi c_{i}^{\delta}}{h} t+\theta_{i}^{\delta}\right)\right) F_{i}^{\delta}$

where $c_{i}^{\delta} \in Z^{+}, a_{i}^{\delta}, b_{i}^{\delta}, \theta_{i}^{\delta} \in \mathfrak{R}$. The function $F_{i}^{\delta}$ is given as follows:

$$
F_{i}^{\delta}=\left[\begin{array}{ccc}
\bar{f}_{11}^{\delta} & \mathrm{L} & \bar{f}_{1 n}^{\delta} \\
\mathrm{M} & \mathrm{O} & \mathrm{M} \\
\bar{f}_{p 1}^{\delta} & \mathrm{L} & \bar{f}_{p n}^{\delta}
\end{array}\right]
$$

where $i=r+(w-1) n$, $w \in\{1,2, \mathrm{~L}, p\}$, $r \in\{1,2, \mathrm{~L}, n\}, \bar{f}_{w r}^{\delta}=1$, and the other term in $F_{i}^{\delta}$ is 0 . In this paper, the delay $\tau$ is considered as $\tau=\frac{h}{m}, m>1$, $m \in Z^{+}$. According to character of delay $\tau$, the model of NCSs can be described as follows:

$$
\begin{aligned}
& x(k+1)=e^{A h} x(k)+ \\
& \sum_{i=1}^{p \times n} \int_{0}^{h-\tau} e^{A s} d s a_{i}^{2} B F_{i}^{2} x(k)+ \\
& \sum_{i=1}^{p \times n} \int_{h-\tau}^{h} e^{A s} d s a_{i}^{1} B F_{i}^{1} x(k-1)+ \\
& \sum_{i=1}^{p \times n} \int_{0}^{h-\tau} e^{A s} d s b_{i}^{2} \sin \left(\frac{2 \pi c_{i}^{2}}{h}+\theta_{i}^{2}\right) B F_{i}^{2} x(k) \\
& \sum_{i=1}^{p \times n} \int_{0}^{h-\tau} e^{A s} d s b_{i}^{1} \sin \left(\frac{2 \pi c_{i}^{1}}{h}+\theta_{i}^{1}\right) B F_{i}^{1} x(k-1)
\end{aligned}
$$

From (6), if the parameter $c_{i}^{\delta} \in Z^{+}$can ensure $\left(I+\frac{h^{2} b^{2}}{\left(2 \pi c^{2}{ }_{i}\right.}\right)^{2} A^{2}$, we have

$\int_{0}^{h-\tau} e^{A s} d s b_{i}^{2} \sin \left(\frac{2 \pi c_{i}^{2}}{h}+\theta_{i}^{2}\right) B F_{i}^{2}$

$=J_{2}(i) \int_{0}^{h-\tau} e^{A s} d s B F_{i}^{2}$

$\int_{h-\tau}^{h} e^{A s} d s b_{i}^{1} \sin \left(\frac{2 \pi c_{i}^{1}}{h}+\theta_{i}^{1}\right) B F_{i}^{1}=$

$J_{1}(i) \int_{h-\tau}^{h} e^{A s} d s B F_{i}^{1}$

where

$J_{2}(i)=\left(I+\frac{h^{2} b_{i}^{2}}{\left(2 \pi c_{i}^{2}\right)^{2}} A^{2}\right)^{-1} \tilde{J}_{2}(i)$
$J_{1}(i)=\left(I+\frac{h^{2} b_{i}^{1}}{\left(2 \pi c_{i}^{1}\right)^{2}} A^{2}\right)^{-1} \tilde{J}_{1}(i)$,

$\tilde{J}_{i}(i)=\sin \left(\theta_{i}^{\prime}\right) \frac{h^{2} b_{i}^{\prime}}{\left(2 \pi c_{i}^{\prime}\right)^{2}} A^{2}-\cos \left(\theta_{i}^{\prime}\right) \frac{h^{2} b_{i}^{\prime}}{2 \pi c_{i}^{\prime}} A$,

$\tilde{J}_{2}(i)=\sin \left(\theta_{i}^{2}\right) \frac{h^{2} b_{i}^{2}}{\left(2 \pi c_{i}^{2}\right)^{2}} A^{2}-\cos \left(\theta_{i}^{2}\right) \frac{h^{2} b_{i}^{2}}{2 \pi c_{i}^{2}} A$

Substituting the (6) and (7) into (5), the model of NCSs can be obtained:

$$
\begin{aligned}
& x(k+1)=e^{A h} x(k)+ \\
& \sum_{i=1}^{p \times n}\left(a_{i}^{2}+J_{2}(i)\right) \int_{0}^{h-\tau} e^{A s} d s B F_{i}^{2} x(k) \\
& +\sum_{i=1}^{p \times n}\left(a_{i}^{1}+J_{1}(i)\right) \int_{h-\tau}^{h} e^{A s} d s B F_{i}^{1} x(k-1)
\end{aligned}
$$

Here it should be mentioned that the initial input $u(t)$ during the interval $(-\infty, \tau]$ is considered as 0 . Then the initial state equation of the (9) can be considered as follows: 


$$
\begin{aligned}
& x(1)=e^{A h} x(0)+ \\
& \sum_{i=1}^{p \times n}\left(a_{i}^{2}+J_{2}(i)\right) \int_{0}^{h-\tau} e^{A s} d s B F_{i}^{2} x(0)
\end{aligned}
$$

Furthermore, in (10), we assume that for any $i$, there exist the following relationship

$\left\|a_{i}^{\delta}+J_{\delta}(i)\right\| \leq \sigma_{i}^{\delta}$

where $\sigma^{\delta}>0$. Correspondingly, if the parameters $\sigma_{i}^{\delta}$ can be determined, the parameters $a_{i}^{\delta}, b_{i}^{\delta}, c_{i}^{\delta}, \theta_{i}^{\delta}$ can be chosen according to (11).

\section{MAIN RESULTS}

In this section, we shall devote to the design of periodic state feedback control laws $F(t)$ for the obtained system (9) with the initial state (10).

Theorem 1. The system (9) with the initial condition (10) is said to be asymptotically stable, if there exist symmetric positive definite matrices $\bar{P}, \quad m \in Z^{+}, \bar{Q}$ $\overline{\mathcal{E}}^{\delta}, \mathcal{E}^{\delta}>0, m>1, i \in\{1,2, \mathrm{~L}, p \times n\}$, the nonsingular matrix $E$, and the approximate dimension matrix satisfying the following LMI:

$$
\begin{aligned}
& {\left[\begin{array}{cccc}
\hat{\Psi}_{11} & \hat{\Psi}_{12} & \hat{\Psi}_{13} & \hat{\Psi}_{14} \\
* & \hat{\Psi}_{22} & 0 & 0 \\
* & * & \hat{\Psi}_{33} & 0 \\
* & * & * & \hat{\Psi}_{44}
\end{array}\right]<0} \\
& \text { where } \hat{\Psi}_{1 "}=\left[\begin{array}{ccc}
\bar{\Omega} & 0 & \left(e^{\text {An }} \mathrm{E}\right)^{T} \\
* & -Q & 0 \\
* & * & -\bar{P}
\end{array}\right] \\
& \bar{\Omega}=\bar{P}+\bar{Q}-E-E^{T}, \hat{\Psi}_{12}=\left[\begin{array}{ccc}
0 & \mathrm{~L} & 0 \\
0 & \mathrm{~L} & 0 \\
\varepsilon_{1}^{\prime} I & \mathrm{~L} & \varepsilon_{p \times n}^{2} I
\end{array}\right] \text {, } \\
& \hat{\Psi}_{22}=\operatorname{diag}\left\{-\varepsilon_{1}^{1} I, \mathrm{~L},-\mathcal{E}_{p \times n}^{2} I\right\}, \\
& \hat{\Psi}_{13}=\left[\hat{Y}^{\tau}(1,1) \cdots \hat{Y}^{\tau}(p \times n, 1)\right] \text {, } \\
& \hat{\Psi}_{33}=\operatorname{diag}\left\{-\overline{\mathcal{E}}_{1}^{1} I, \mathrm{~L},-\overline{\mathcal{E}}_{p \times n}^{1} I\right\}, \\
& \hat{\Psi}_{14}=\left[\tilde{Y}^{T}(1,2) \cdots \tilde{Y}^{T}(p \times n, 2)\right],
\end{aligned}
$$

$$
\begin{aligned}
& \hat{\Psi}_{44}=\operatorname{diag}\left\{-\bar{\varepsilon}_{1}^{2} I, \mathrm{~L},-\bar{\varepsilon}_{p \times n}^{2} I\right\}, \\
& \hat{Y}(i, \delta)=\left[\begin{array}{llll}
0 & \int_{h-\tau}^{h} e^{A s} d s B F_{i}^{\delta} E & 0
\end{array}\right], \\
& \tilde{Y}(i, \delta)=\left[\begin{array}{lll}
\int_{0}^{h-\tau} e^{A s} d s B F_{i}^{\delta} E & 0 & 0
\end{array}\right] .
\end{aligned}
$$

Moreover, the parameters $\sigma_{i}^{\delta}$ can be calculated by using $\sigma_{i}^{\delta}=\sqrt{\frac{\varepsilon_{i}^{\delta}}{\bar{\varepsilon}_{i}^{\delta}}}$. Based on this condition, the parameters $b_{i}^{\delta}, c_{i}^{\delta}, \theta_{i}^{\delta}, a_{i}^{\delta}$ can be chosen according to (11). Correspondingly, the NTPPF $F(t)$ can be designed.

Proof: At first, the Lyapunov function is taken as

$$
V(k)=x^{T}(k) P x(k)+x^{T}(k-1) Q x(k-1)
$$

where $k=k h$.Then, we have

$$
\begin{aligned}
& V(k+1)=x^{T}(k+1) \operatorname{Px}(k+1) \\
& +x^{T}(k) Q x(k)
\end{aligned}
$$

It is obvious that

$$
\begin{aligned}
& \Delta V=V(k+1)-V(k) \\
& =\left[\begin{array}{c}
x(k) \\
x(k-1)
\end{array}\right]^{T}\left[\begin{array}{cc}
\Xi_{11} & \Xi_{12} \\
* & \Xi_{22}
\end{array}\right]\left[\begin{array}{c}
x(k) \\
x(k-1)
\end{array}\right]
\end{aligned}
$$

where $\Xi_{11}=-P+Q+U^{T} P\left(l_{k+1}\right) U, \Xi_{12}=U^{T} P\left(l_{k+1}\right) W$, $U=e^{A h}+\sum_{i=1}^{p \times n}\left(a_{i}^{2}+J_{2}(i)\right) \int_{0}^{h-\tau} e^{A s} d s B F_{i}^{2}, \quad \Xi_{22}=W^{T} P W$, $W=\sum_{i=1}^{p \times n}\left(a_{i}^{1}+J_{1}(i)\right) \int_{h-\tau}^{h} e^{A s} d s B F_{i}^{1}$ If the inequality $\left[\begin{array}{cc}\Xi_{11} & \Xi_{12} \\ * & \Xi_{22}\end{array}\right]<0$ holds, we have $V(k+1)<V(k)$ for any $x(k) \neq 0$ and $x(k-1) \neq 0$, which means $\lim V(k)=0$. By using Schur complement Lemma, the equality $\left[\begin{array}{cc}\Xi_{11} & \Xi_{12} \\ * & \Xi_{22}\end{array}\right]<0$ can be described as 
$\left[\begin{array}{ccc}-P+Q & 0 & U^{T} \\ * & -Q & W^{T} \\ * & * & -P^{-1}\end{array}\right]<0$

Furthermore, we have

$\left[\begin{array}{ccc}-P+Q & 0 & \left(e^{4 h}\right)^{T} \\ * & -Q & 0 \\ * & * & -P^{-1}\end{array}\right]+$

$\sum_{i=1}^{p \times n} \operatorname{sym}\left\{\left[\begin{array}{l}0 \\ 0 \\ I\end{array}\right]\left(a_{i}^{1}+J_{1}^{i}\right) G(i, 1)\right\}$

$+\sum_{i=1}^{p \times n} \operatorname{sym}\left\{\left[\begin{array}{l}0 \\ 0 \\ I\end{array}\right]\left(a_{i}^{2}+J_{1}^{2}\right) \bar{G}(i, 2)\right\}<0$,

where $G(i, \delta)=\left[\begin{array}{lll}0 & \int_{h-\tau}^{h} e^{4 s} d s B F_{i}^{\delta} & 0\end{array}\right]$,

$\bar{G}(i, \delta)=\left[\int_{0}^{h-\tau} e^{4 \delta} d s B F_{i}^{\delta} \quad 0 \quad 0\right]$.

Moreover, we can obtain

$\sum_{i=1}^{p \times n} \operatorname{sym}\left\{\left[\begin{array}{l}0 \\ 0 \\ I\end{array}\right]\left(a_{i}^{i}+J_{i}^{i}\right) G(i, 1)\right\} \leq$

$\sum_{i=1}^{p \times n}\left(\varepsilon_{i}^{i}\left[\begin{array}{l}0 \\ 0 \\ I\end{array}\right]\left[\begin{array}{l}0 \\ 0 \\ I\end{array}\right]^{T}\right)+$

$\sum_{i=1}^{p \times n}\left(\frac{\left(\sigma_{i}^{1}\right)^{2}}{\varepsilon_{i}^{1}} G^{T}(i, 1) G(i, 1)\right)$

and

$$
\begin{aligned}
& \sum_{i=1}^{p \times n} \operatorname{sym}\left\{\left[\begin{array}{l}
0 \\
0 \\
I
\end{array}\right]\left(a_{i}^{2}+J_{2}^{i}\right) G(i, 2)\right\} \leq \\
& \sum_{i=1}^{p \times n}\left(\varepsilon_{i}^{2}\left[\begin{array}{l}
0 \\
0 \\
I
\end{array}\right]\left[\begin{array}{l}
0 \\
0 \\
I
\end{array}\right]^{T}\right)+ \\
& \sum_{i=1}^{p \times n}\left(\frac{\left(\sigma_{i}^{2}\right)^{2}}{\varepsilon_{i}^{2}} \bar{G}^{T}(i, 2) \bar{G}(i, 2)\right) \\
& \text { for any } \varepsilon_{i}^{\delta}>0 \text {. }
\end{aligned}
$$

By using the schur complement Lemma, we have

$$
\left[\begin{array}{cccc}
\Psi_{11} & \Psi_{12} & \Psi_{13} & \Psi_{14} \\
* & \Psi_{22} & 0 & 0 \\
* & * & \hat{\Psi}_{33} & 0 \\
* & * & * & \hat{\Psi}_{44}
\end{array}\right]<0
$$

where $\quad \Psi_{11}=\left[\begin{array}{ccc}-P+Q & 0 & \left(e^{4 h}\right)^{T} \\ * & -Q & 0 \\ * & * & -P^{-1}\end{array}\right]$,

$\Psi_{12}=\left[\begin{array}{ccc}0 & \mathrm{~L} & 0 \\ 0 & \mathrm{~L} & 0 \\ I & \mathrm{~L} & I\end{array}\right]$,

$\Psi_{13}=\left[G^{T}(1,1) \mathrm{L} G^{T}(p \times n, 1)\right]$,

$\Psi_{22}=\operatorname{diag}\left\{-\frac{1}{\varepsilon_{1}^{\prime}} I, \mathrm{~L}-\frac{1}{\varepsilon_{p \times n}^{2}} I\right\}$,

$\Psi_{14}=\left[\bar{G}^{T}(1,2) \mathrm{L} \bar{G}^{T}(p \times n, 2)\right] \cdot$ Here for any nonsingular matrix $E \in \mathfrak{R}^{n \times n}$, we use the matrix $E^{-T} P^{-1} E^{-1}-E^{-1}-E^{-T}+Q$ to replace the matrix $-P+Q$ in (20). Let $\bar{P}=P^{-1}, \bar{Q}=E^{T} Q E$, we can obtain the (12) by pre- and post-multiplying the matrix $\operatorname{diag}\left\{E^{T}, E^{T}, \tilde{\Omega}, I\right\}$ and its transpose matrix, where $\tilde{\Omega}=\operatorname{diag}\left\{\varepsilon_{1}^{1} I, \cdots, \varepsilon_{p \times n}^{2} I\right\}$. This completes the proof. 


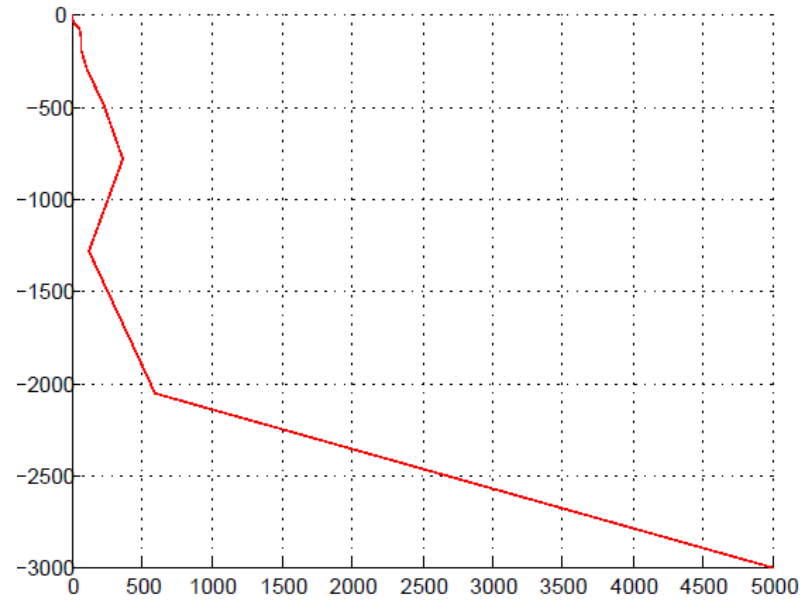

Fig. (1). State response curve of NCSs (21).

\section{NUMERICAL EXAMPLE}

In this section, we present a numerical simulation to show the application of the proposed methods in this paper. Considering the continuous-time linear time-invariant model as

$\dot{x}(t)=\left[\begin{array}{cc}0 & 1 \\ 0 & -0.1\end{array}\right] x(t)+\left[\begin{array}{c}0 \\ 0.1\end{array}\right] u(t)$

The sampling period $h=5 \mathrm{~s}, \tau=2.5 \mathrm{~s}$. Then we can obtain the following results by using Theorem 1: $\sigma_{1}^{1}=0.59$, $\sigma_{1}^{2}=0.56, \sigma_{2}^{1}=0.58, \sigma_{2}^{2}=0.56$. Furthermore, the parameters of NTPPF can be chosen as: $a_{1}^{1}=4.2 \times 10^{-5}$, $a_{2}^{1}=-7.1 \times 10^{-3}, a_{1}^{2}=-3.1, a_{2}^{2}=-11.175, \quad c_{1}^{1}=10$, $c_{1}^{2}=5, c_{2}^{1}=5, c_{2}^{2}=15, b_{1}^{1}=0.1, b_{1}^{2}=0.13, b_{2}^{1}=0.05$, $b_{2}^{2}=1.25, \theta_{1}^{1}=\theta_{2}^{1}=\pi / 4, \theta_{1}^{2}=\theta_{2}^{2}=\pi / 3$.

According to the obtained parameters, the state response curves under the case of initial conditions $x(0)=$ $\left[\begin{array}{ll}5000 & -3000\end{array}\right], x(0)=\left[\begin{array}{ll}600 & -3\end{array}\right]$ and $x(0)=\left[\begin{array}{ll}50 & -320\end{array}\right]$ are plotted in Figs. (1-3). It shows that the designed GSHF for NCSs works well. Therefore, according to the analysis mentioned above, the results show that the effective and practical value of our methods.

\section{CONCLUSION}

In this paper, the design problem of NCSs based on multi-rata technique has been considered. The obtained results can be considered as the development of the theory in [9], which only considered the special case of GSHF. A novel NTPPF method which is based on the Fourier series of periodic signal, has been proposed to construct the GSHF. Then, a hybrid nonlinear delay system model for NCSs has been

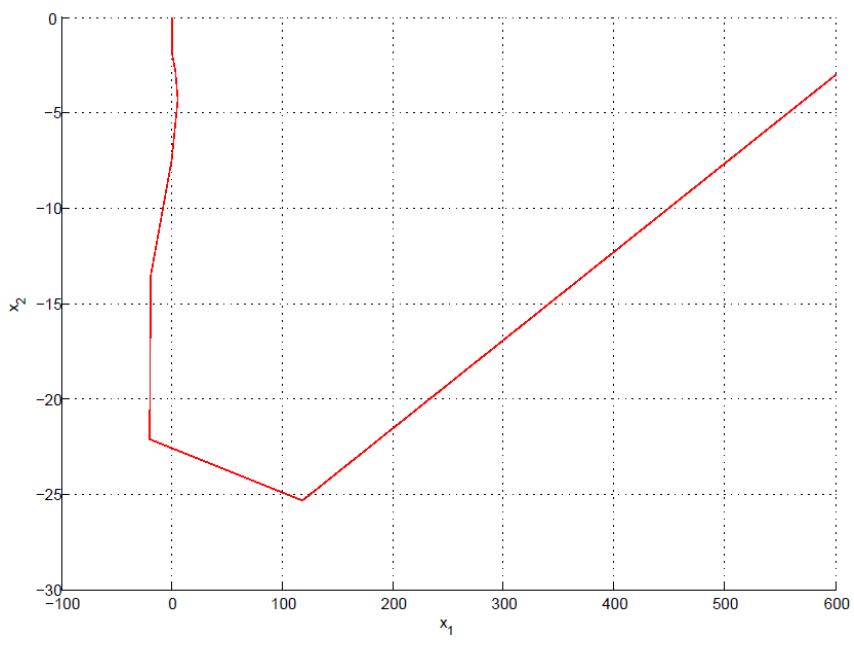

Fig. (2). State response curve of NCSs (21).

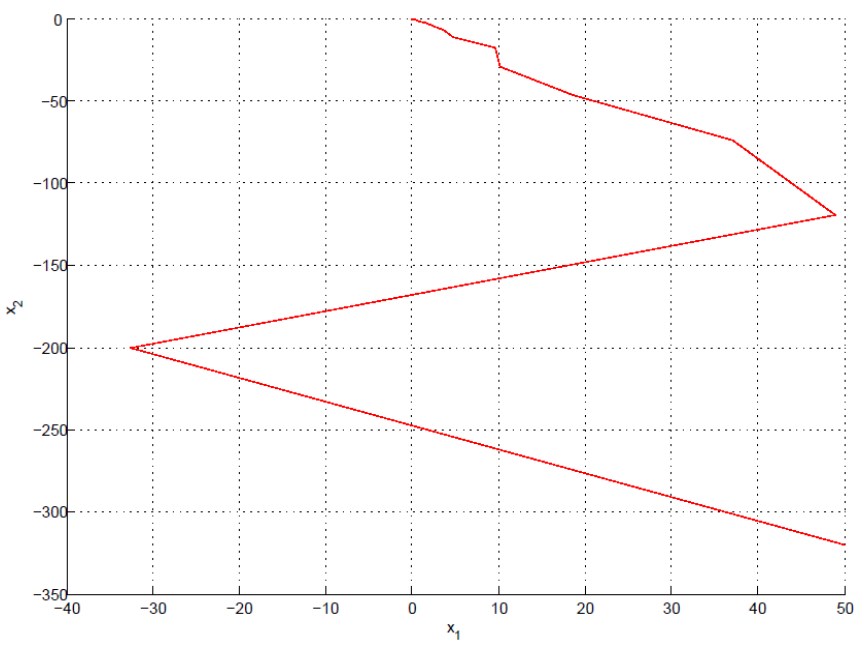

Fig. (3). State response curve of NCSs (21).

modeled. Furthermore, the stability conditions were obtained by using the Lyapunov theory. And the NTPPF has been designed for ensuring the stability of NCSs. Finally, a numerical example has been provided to demonstrate the effectiveness of the proposed methods.

\section{CONFLICT OF INTEREST}

The authors confirm that this article content has no conflict of interest.

\section{ACKNOWLEDGEMENTS}

This work is supported by the China Postdoctoral Science Foundation Funded Project (2014M551110) and National Natural Science Foundation of China (61403073).

\section{REFERENCES}

H.G. Zhang, D.D. Yang, and T.Y. Chai, "Guaranteed cost networked control for T-S fuzzy systems with time delays", IEEE Transactions on Systems, Man, and Cybernetics-Part C: Applications and reviews, vol. 37, pp. 160-172, 2007. 
[2] W.A. Zhang, and L. Yu, "A robust control approach to stabilization of networked control systems with time-varying delays", Automatica, vol. 45, pp. 2440-2445, 2009.

[3] H. G. Zhang, M. Li, J. Yang, and D. D. Yang, "Fuzzy modelbased robust networked control for a class of nonlinear systems", IEEE Transactions on Systems, Man, and Cybernetics--Part A: Systems and Humans, vol. 39, pp. 437-447, 2009.

[4] H. G. Zhang, J. Yang and C. Y. Su, "T-S fuzzy-model-based robust H-infinite design for networked control systems with uncertainties", IEEE Transactions on Industrial Informatics, vol. 3, pp. 289301, 2007.

[5] P. T. Kabamba, "Control of linear systems using generalized sampled-data hold function", IEEE Transactions on Automatic Control, vol. AC-32, no. 9, pp. 772-783, 1987.

[6] M. B. Naumovic, and M. R. Stojic, "Generalized sampled-hold functions approach to filtering and control", $5^{\text {th }}$ International Conference on Telecommunications in Modern Satellite, Cable and Broadcasting Service, pp. 712-793, 2001.

[7] J. Lavaei, S. Sojoudi, and A. G. Aghdam, "Pole assignment with improved control performance by means of periodic feedback", IEEE Transactions on Automatic Control, vol. 55, pp. 248-252, 2010.

[8] A. Feuer, and G. C. Goodwin, "Generalized sample hold functions: Frequency domain analysis of robustness, sensitivity, and inter- sample difficulties", IEEE Transactions on Automatic Control, vol. 39, pp. 1042-1047, 1995.

[9] H. G. Zhang, G. T. Hui, and Y. C. Wang, "Stabilization of networked control systems with piecewise constant generalized sampled-data hold function", International Journal of Innovative Computing, Information and Control. vol. 9, pp. 1159-1170, 2013.

[10] J. Lavaei, and A. G. Aghdam, "Overlapping control design for multi-channel systems", Automatica, vol. 45, pp. 1326-1331, 2009.

[11] H. G. Zhang, Z. S. Wang, and D. R. Liu, "A comprehensive review of stability analysis of continuous-time recurrent neural networks", IEEE Trans on Neural Networks and Learning Systems, vol. 25, 1229-1262, 2014.

[12] H. G. Zhang, C. B. Qing, B. Jiang, and Y.H. Luo, "Online adaptive policy learning algorithm for $\mathrm{H}$-infinite state feedback control of unknown affine nonlinear discrete-time systems", IEEE Transactions on Cybernetics, vol. 44, pp. 2706-2718, 2014.

[13] H. G. Zhang, F. S. Yang, X. D. Liu, and Q. L. Zhang, "Stability analysis for neural networks with time-varying delay based on quadratic convex combination", IEEE Trans on Neural Networks and Learning Systems, vol. 24, pp. 513-521, 2013.

[14] G. T. Hui, H. G. Zhang, Z. N. Wu, Y. C. Wang, "Control synthesis problem for networked linear sampled-data control systems with bandlimited channels", Information Sciences, vol. 375, pp. 385399, 2014.

Received: May 26, 2015

Revised: July 14, 2015

Accepted: August 10, 2015

(C) Hui et al.; Licensee Bentham Open.

This is an open access articles licensed under the terms of the Creative Commons Attribution-Non-Commercial 4.0 International Public License (CC BY-NC 4.0) (https://creativecommons.org/licenses/by-nc/4.0/legalcode), which permits unrestricted, non-commercial use, distribution and reproduction in any medium, provided that the work is properly cited. 